九州大学学術情報リポジトリ

Kyushu University Institutional Repository

\title{
System Identification and Adaptive Control of Mass-Varying Quad-Rotor
}

Dief, Tarek N.

Interdisciplinary Graduate School of Engineering Sciences, Kyushu University

Yoshida, Shigeo

Interdisciplinary Graduate School of Engineering Sciences, Kyushu University

https://doi.org/10.5109/1808454

出版情報: Evergreen. 4 (1)，pp.58-66，2017-03. Green Asia Education Center バージョン :

権利関係 : 


\title{
System Identification and Adaptive Control of Mass-Varying Quad-Rotor
}

\author{
Tarek N. Dief*, Shigeo Yoshida \\ Interdisciplinary Graduate School of Engineering Sciences, Kyushu University, Japan \\ *Author to whom correspondence should be addressed, \\ E-mail: tarek.na3em@riam.kyushu-u.ac.jp
}

(Received January 8, 2017; accepted March 1, 2017).

\begin{abstract}
Modelling, system identification and the controller for variable length quad-rotors are presented in this paper. Modelling was derived using Newton-Euler method, then small disturbance theory was used for linearization. Plackett's algorithm was used for system identification to predict the system parameters. Mass-varying problem is the main objective of this paper, and the effect of changing the system parameters was discussed in detail. The system parameters are updated in real-time during flight with low sample time. The PID-Accelerator controller of the quad-rotor was updated also in real-time based on the change of the system identification output. The attitude and altitude control of the quad-rotor were presented using an adaptive classical controller. Now the tethered mass-varying quad-rotor can be simulated. The objective of this work is to make the quad rotors fly for long time and to be robust for variable inputs comes from the tether. The simulation results of the system identification and control responses of the attitude and altitude are presented in this paper. The disturbance of the wind was also considered in the controller design.
\end{abstract}

Keywords: System Identification, Plackett’s algorithm, Quad-Rotor, PID-A controller.

\section{Nomenclature}

\begin{tabular}{|c|c|c|}
\hline$b$ & C & Thrust constant of the rotor. \\
\hline$d$ & & Torque constant of the rotor. \\
\hline$m$ & & System total mass \\
\hline$I_{r}$ & & Rotor inertia around the Rotor axis. \\
\hline$I_{x}, I_{y}, I_{z}$ & & $\begin{array}{l}\text { Moment of inertia }\left(\mathrm{x}^{-}, \mathrm{y}^{-} \text {, and } \mathrm{z}^{-} \text {-axis }\right) \text { of } \\
\text { quad-rotor. }\end{array}$ \\
\hline$L$ & & $\begin{array}{l}\text { Length between the motor center and the } \\
\text { body center of gravity. }\end{array}$ \\
\hline$P_{k}$ & & $\begin{array}{l}\text { Covariance matrix of the estimated error, and } \\
\text { it presents how this system true or not by } \\
\text { calculating this matrix value. }\end{array}$ \\
\hline$p, q, r$ & & $\begin{array}{l}\text { Rotational velocity in body frame around } \mathrm{i}, \mathrm{j} \text {, } \\
\mathrm{k} \text {. }\end{array}$ \\
\hline$R$ & $\theta^{\circ}$ & Rotation matrix. \\
\hline$s$ & & Laplace operator. \\
\hline$T$ & & Sample time. \\
\hline$u, v, W$ & & $\begin{array}{l}\text { Translational velocity in body frame along } \mathrm{i}, \mathrm{j} \text {, } \\
\mathrm{k} \text { frame w.r.t inertia frame. }\end{array}$ \\
\hline$V$ & & Translational velocity vector. \\
\hline$X_{k}$ & & $\begin{array}{l}\text { Vector contains the present and the past } \\
\text { values of the output } y \text { and input } u \text {. }\end{array}$ \\
\hline $\mathbf{M}_{\mathbf{b}}$ & & Moment vector in body axes. \\
\hline$X$ & & $\begin{array}{l}\text { Positions vector of the body frame w.r.t } \\
\text { inertia frame. }\end{array}$ \\
\hline$Y$ & & Estimated model output. \\
\hline$Y_{m}$ & & $\begin{array}{l}\text { Actual model output that can be obtained } \\
\text { from the sensors. }\end{array}$ \\
\hline & & Position of the body frame w.r.t inertia \\
\hline
\end{tabular}

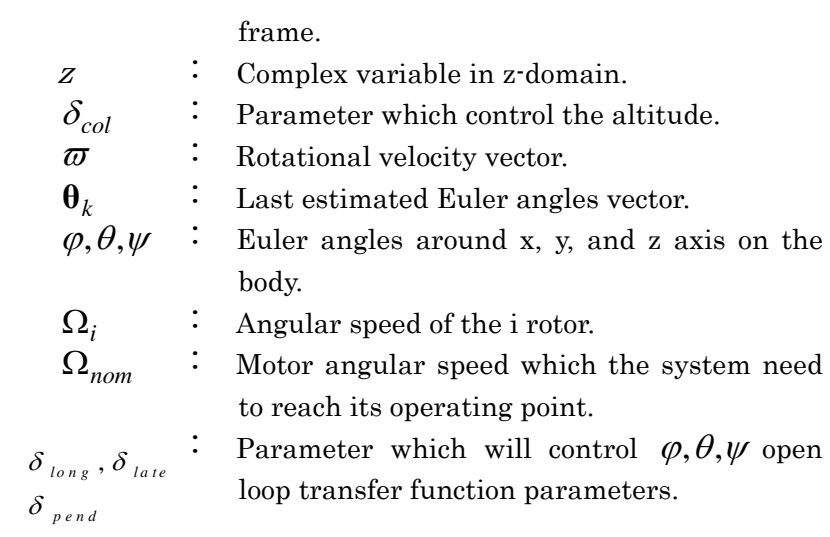

\section{Introduction}

Small Unmanned Aerial Vehicles (UAVs) are being used widely in research and civil applications such as aerial photography and inspection. Recent developments in Micro Electro-Mechanical Systems (MEMS) opened the door for the civil applications ${ }^{1-3}$.

Helicopter aircrafts have advantages in taking-off, landing vertically and hovering at the same position for long time. Thus, they can achieve some missions not available for the fixed wing UAVs. On the other hand, the helicopter systems use swashplates for stabilization, these swashplates are mechanically complex, not available for commercial usage in different sizes, and expensive. So, the design of the quad-rotor can avoid 
these difficulties in the helicopter system with achieving the same mission. Several papers have been written regarding modelling and controlling quad-rotors with consideration of fixing the parameters of the system ${ }^{4-9)}$.

In spite of the achievements made in this field, scholars are still facing different problems regarding the large payloads and long flight-times. Some researchers studied the problem of unmanned quad-rotor helicopter connected with cable to the ground. This kind of cable has a variable length and payload, and this cable is electrified to transmit the power to the quad-rotor. The field of tethered aerostats and tethered space satellites are usually using the cable connections. Also, other researches derived mathematical model for the tether connected to the quad-rotor, and tried to give fully aerodynamic analysis for its motion.

Deng et al., applied wind load to balloon and showed its effect to the elastic mooring rope tension, this tests were applied to the high altitude balloons ${ }^{10)}$. Other research applied the concept of using the damping spring system to simulate the ascent sequence for the tethered balloon $^{11)}$. The idea of connecting the tether to the quad-rotor and modify the mathematical model based on this editing was discussed in detail, the aim for this cable is to supply power to the quad-rotor instead of batteries, the controller used to stabilize the quad-rotor was classical PID control ${ }^{12)}$. Other research mentioned the effect for the tether connected to the ground, and new stabilization algorithm was applied to recover the vehicle's relative position and absolute oriention ${ }^{13)}$.

The proposed method requires only on-board inertial sensors and indirectly measures the string force, enabling the additional use of the tether as a physical user interaction medium. The mathematical model of the tether and the quad-rotor are not totally accurate or close to the real behavior of the tethered quad-rotor ${ }^{17-19)}$. So choosing the system prediction or identification is one of the promising ideas for the tethered quad-rotors. Using this algorithm will predict the system governing equations based on the sensors readings.

In this paper, the mathematical model of the quad-rotor with variable-length tether was presented after considering the quad-rotor and the tether move in fixed 2-D plane, and the quad-rotor is represented as a point mass at the end of the straight tether of variable length during the whole flight. The governing equations including the nonlinear equations followed with the linearization was derived. The linearized model was derived for Single-Input Single-Output (SISO) and the small disturbance theory to linearize the model. Plackett's algorithm concept ${ }^{5,15)}$ was used for the system identification to generate the system parameters.

Then, the adaptive controller is used to stabilize the quad-rotor in real-time based on the updated parameters of the system identification. The simulations for the system after controller is presented during the change of the tether length. Moreover, this paper get description for the quad-rotor's motion after connecting it with tether. The simulation for the quad-rotors during for a long time and also flying considering the effect of tether in real-time were presented in this paper. Also robust controller to stabilize the tethered quad-rotor under any disturbance that might happen were discussed in detail.

\section{Modelling \& Linearization}

The quad-rotor problem is basically nonlinear, multi-input multi-output (MIMO), and under actuated system with six degrees of freedom for just four actuators. Some assumptions were considered to simplify the derivation of the mathematical model of the quad-rotor. These assumptions considered the quad-rotor as a symmetric body with a rigid frame armed with four rotors. The weight of the quad-rotor doesn't change during flight (i.e. time-invariant). The center of gravity (COG) is fixed at the origin of the quad-rotor. The thrust and drag constants are proportional to the square value of the motor's speed.

The model of the quad-rotor is given in ${ }^{14)}$, and the parameters used for designing the quad-rotor are given in Table 1.

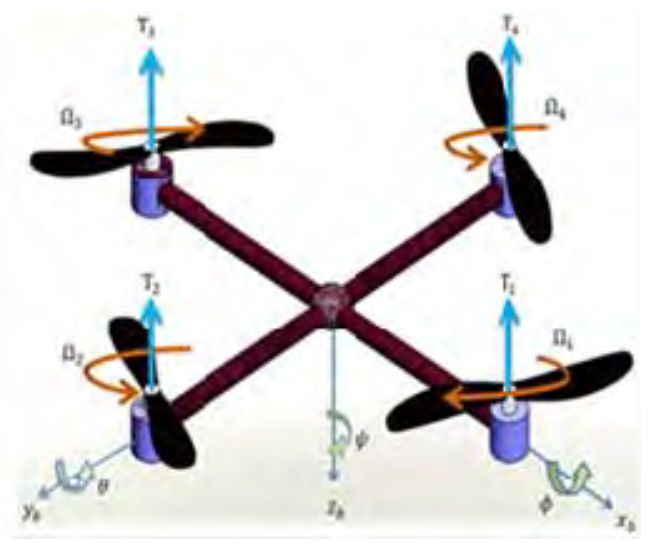

Fig. 1. Quad-rotor with body axes.

The linearized Equations 3-6 were derived using small disturbance theory ${ }^{14)}$. The initial conditions that were used for the derivations are:

$$
\phi_{o}=\theta_{o}=\psi_{o}=\dot{\phi}_{o}=\dot{\theta}_{o}=\dot{\psi}_{o}=0
$$$$
u_{o}=v_{o}=w_{o}=p_{o}=q_{o}=r_{o}=0
$$

Equations (1-2) are used to get the nonlinear model of the quad-rotor using Euler angles as following: 


$$
\begin{aligned}
& {\left[\begin{array}{c}
\dot{U} \\
\dot{V} \\
\dot{W}
\end{array}\right]=\left[\begin{array}{c}
-g \sin \theta+q w-v r \\
g \cos \theta \sin \varphi-p w+u r \\
-\frac{U_{1}}{m}+g \cos \theta \cos \varphi+p v-q u
\end{array}\right]} \\
& {\left[\begin{array}{c}
\dot{\varphi} \\
\dot{\theta} \\
\dot{\psi}
\end{array}\right]=\left[\begin{array}{ccc}
1 & \tan \theta \sin \varphi & \tan \theta \cos \varphi \\
0 & \cos \varphi & -\sin \varphi \\
0 & \frac{\sin \varphi}{\cos \theta} & \frac{\cos \varphi}{\cos \theta}
\end{array}\right]\left[\begin{array}{l}
p \\
q \\
r
\end{array}\right]}
\end{aligned}
$$

Then, the linear open loop transfer functions would be:

$$
\begin{aligned}
& \frac{\varphi}{\delta_{\text {late }}}=\frac{2 b l \Omega_{n o m}}{I_{x x} s^{2}} \\
& \frac{\theta}{\delta_{\text {long }}}=\frac{2 b l \Omega_{n o m}}{I_{y y} s^{2}} \\
& \frac{\psi}{\delta_{\text {pend }}}=\frac{2 d \Omega_{\text {nom }}}{I_{z z} s^{2}} \\
& \frac{z}{\delta_{\text {col }}}=\frac{-2 b \Omega_{\text {nom }}}{m s^{2}}
\end{aligned}
$$

Table 1. System parameters.

\begin{tabular}{|c|c|}
\hline Parameters (unit) & Value \\
\hline$m(\mathrm{~kg})$ & 2 \\
\hline$g\left(\mathrm{~m} / \mathrm{s}^{2}\right)$ & 9.81 \\
\hline$b\left(\mathrm{NS}^{2}\right)$ & $9.6^{*} 10^{-6}$ \\
\hline$d\left(\mathrm{Ns}^{2}\right)$ & $1.24^{*} 10^{-7}$ \\
\hline$L(\mathrm{~m})$ & 0.25 \\
\hline$I_{x x}=I_{y y}\left(\mathrm{kgm}^{2}\right)$ & $8.945^{*} 10^{-3}$ \\
\hline$I_{z z}\left(\mathrm{kgm}^{2}\right)$ & 0.0165 \\
\hline$I_{r}\left(\mathrm{kgm}^{2}\right)$ & $1.28^{*} 10^{-4}$ \\
\hline
\end{tabular}

\section{System Identification Using Placket's algorithm}

In the previous section, the model was considered fixed and there was no change in the system's mass. For this section, it is required to show the effect of mass variation of the system during flight. Thus, it is needed to update the parameters in real-time by knowing the history of the model's inputs and outputs data. Least square estimation was used as a system identification to update the system's governing equations. The aim of this model is to get the system parameters at the minimum Mean Square Error MSE as shown in Equation (7).

$$
M S E=\frac{1}{k} \sum_{r=1}^{k}\left(Y(r)-Y_{m}(r)\right)^{2}
$$

Where, $r$ is number of the time step in the discrete time process. The open loop transfer function of the quad-rotor is derived under the assumption of no change in system parameters, thus the mathematical model can't define the system exactly. This uncertainty in the mathematical model will occur due to the variation of inertia, mass, or motor's constants. So we can use the parameter estimation to get updated values for the open loop transfer function as shown in fig. 2.

This Recursive Least Square Estimation (RLSE) used in this paper is called Plackett's algorithm. This model has the advantages of getting the accurate values for system parameters so fast and has no singularity for any initial conditions even they are zeros ${ }^{15)}$.

$$
\dot{\omega}=\left[\begin{array}{c}
\dot{p} \\
\dot{q} \\
\dot{r}
\end{array}\right]=\left[\begin{array}{l}
\frac{\left(I_{y y}-I_{z z}\right) q r+U_{2}}{I_{x x}} \\
\frac{\left(I_{z z}-I_{x x}\right) p r+U_{3}}{I_{y y}} \\
\frac{\left(I_{x x}-I_{y y}\right) p q+U_{4}}{I_{z z}}
\end{array}\right]
$$

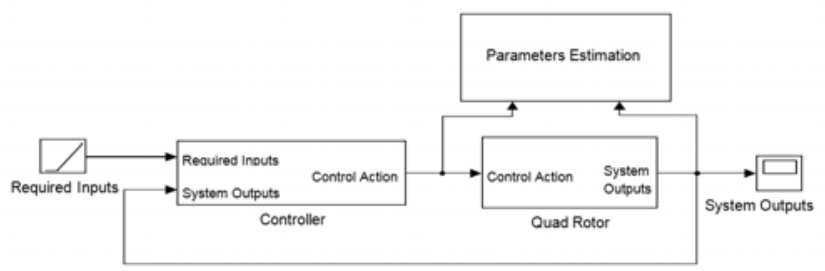

Fig. 2. Block diagram of the adaptive control model.

Plackett's algorithm uses the motor action and the sensor data to estimate the system's parameters. The open loop transfer function of the quad-rotor can be written as a function of the polynomials $A\left(z^{-1}\right)$ and $B\left(z^{-1}\right)$ as a shown in Equation (10):

$$
G\left(z^{-1}\right)=\frac{Y\left(z^{-1}\right)}{U\left(z^{-1}\right)}=\frac{B\left(z^{-1}\right)}{A\left(z^{-1}\right)}
$$

$A\left(z^{-1}\right)$ and $B\left(z^{-1}\right)$ are considered as a second order polynomial in z-domain. After rewriting $A\left(z^{-1}\right)$ and $B\left(z^{-1}\right)$ in the discrete form, they will be as following in Equations (11-12) 


$$
\begin{gathered}
A\left(z^{-1}\right)=1+a_{1} z^{-1}+a_{2} z^{-2} \\
B\left(z^{-1}\right)=b_{1} z^{-1}+b_{2} z^{-2}
\end{gathered}
$$
(10):

After substituting by Equations (11-12) into Equation

$$
\frac{Y}{U}\left(z^{-1}\right)=\frac{b_{1} z^{-1}+b_{2} z^{-2}}{1+a_{1} z^{-1}+a_{2} z^{-2}}
$$

Also we can rewrite Equation (13) in different form as shown in Equation (14):

$$
\begin{array}{r}
Y(k)=-a_{1} Y(k-1)-a_{2} Y(k-2)+b_{1} U(k-1) \\
+b_{2} U(k-2)
\end{array}
$$

First, the open loop model Equation (14) can be rewritten as shown in Equation (15):

$$
Y(k)=\mathbf{X}_{k-1}^{T} \boldsymbol{\theta}_{k-1}
$$

Where:

$$
\boldsymbol{\theta}_{k-1}=\left[\begin{array}{c}
-a_{1} \\
-a_{2} \\
b_{1} \\
b_{2}
\end{array}\right], \quad \mathbf{X}_{k-1}=\left[\begin{array}{c}
Y(k-1) \\
Y(k-2) \\
U(k-1) \\
U(k-2)
\end{array}\right]
$$

After rewriting the MSE between actual model and estimated model can be written as following:

$$
\text { MSE }=\frac{1}{n} \sum_{k=1}^{n}\left(\mathbf{X}_{k-1}^{T} \boldsymbol{\theta}_{k-1}-\mathbf{Y}_{m}(k)\right)^{2}
$$

The objective of using the system identification is to get the values of $\boldsymbol{\theta}_{k}$ that can minimize the mean square error. From the derivation, the values of $\boldsymbol{\theta}_{k}$ can be easily calculated from Equation (16):

$$
\boldsymbol{\theta}_{k}=\mathbf{P}_{k}\left[\sum_{k=1}^{n}\left(\mathbf{X}_{k-1} \mathbf{Y}(k)\right)\right]
$$

Where:

$$
\mathbf{P}_{k}=\left[\sum_{k=1}^{n}\left(\mathbf{X}_{k-1} \mathbf{X}_{k-1}^{T}\right)\right]^{-1}
$$

By rewriting Equation (17) we can find the following Equation (18):

$$
\mathbf{P}_{k}=\mathbf{P}_{k-1}-\frac{\mathbf{P}_{k-1} \mathbf{X}_{k} \mathbf{X}_{k}^{T} \mathbf{P}_{k-1}}{1+\mathbf{X}_{k}^{T} \mathbf{P}_{k-1} \mathbf{X}_{k}}
$$

$$
\boldsymbol{\theta}_{k}=\boldsymbol{\theta}_{k-1}-\frac{\mathbf{P}_{k-1} \mathbf{X}_{k}}{1+\mathbf{X}_{k}^{T} \mathbf{P}_{k-1} \mathbf{X}_{k}}\left(\mathbf{X}_{k}^{T} \boldsymbol{\theta}_{k-1}-Y(k)\right)
$$

The open loop transfer functions of the attitude and altitude are given in Equations (16-19), and they were rewritten in z-domain using difference form given in Equation (14). Thus, the parameters $a_{1}, a_{2}, b_{1}$ and $b_{2}$ were updated at every time step for roll, pitch, yaw, and altitude.

To get theses parameters, it is needed to go through the following steps of calculations:

Initialize matrix $\mathbf{P}_{k-1}$ with large positive numbers on the leading diagonal and zeros on the off diagonal elements.

Initialize vector $\boldsymbol{\theta}_{k-1}$ with parameters close to the model.

1. $\mathbf{X}_{k}$ is updated every sample time by system outputs and inputs as defined before.

2. Calculate $\boldsymbol{\theta}_{k}$ and $\mathbf{P}_{k}$ from Equations (18) \& (19).

3. Update $\boldsymbol{\theta}_{k-1} \& \mathbf{P}_{k-1}$ with $\boldsymbol{\theta}_{k} \& \mathbf{P}_{k}$.

Repeat the loop for each time step.

After applying this algorithm for the attitude and altitude, we can get the following results in Figs. 3-10. White noise was added to the system with amplitude \pm 0.5 degree, and the simulation was achieved using the linearized model.

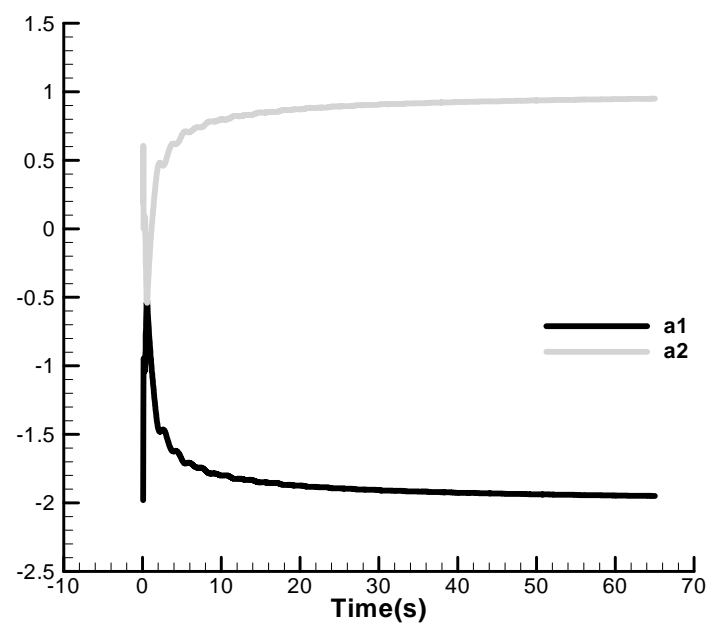

Fig. 3. Time history of $a_{1}$ and $a_{2}$ for roll.

In Fig. 3, the values of $a_{1}$, and $a_{2}$ are updated in real time for roll motion. 


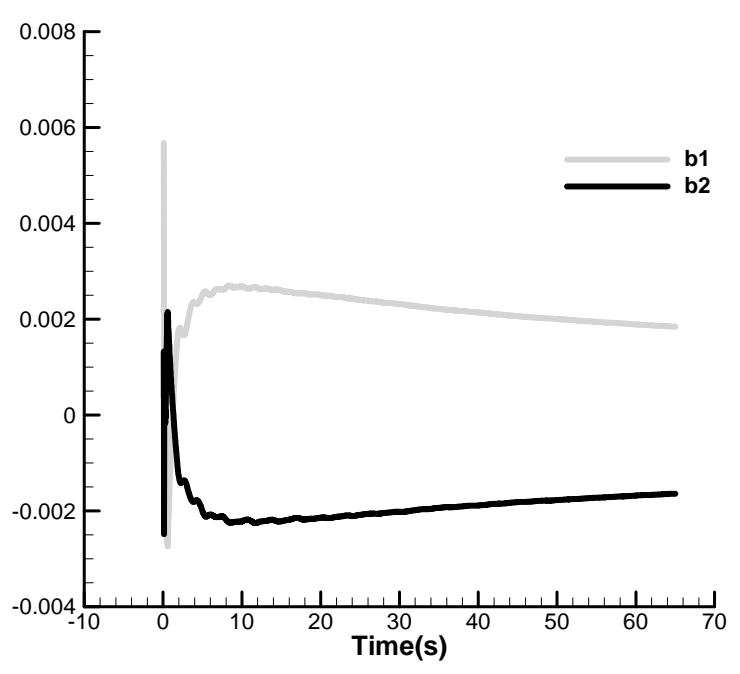

Fig. 4. Time history of $b_{1}$ and $b_{2}$ for roll.

In Fig. 4 , the values of $b_{1}$, and $b_{2}$ are calculated from equation 19 in real time for roll motion.

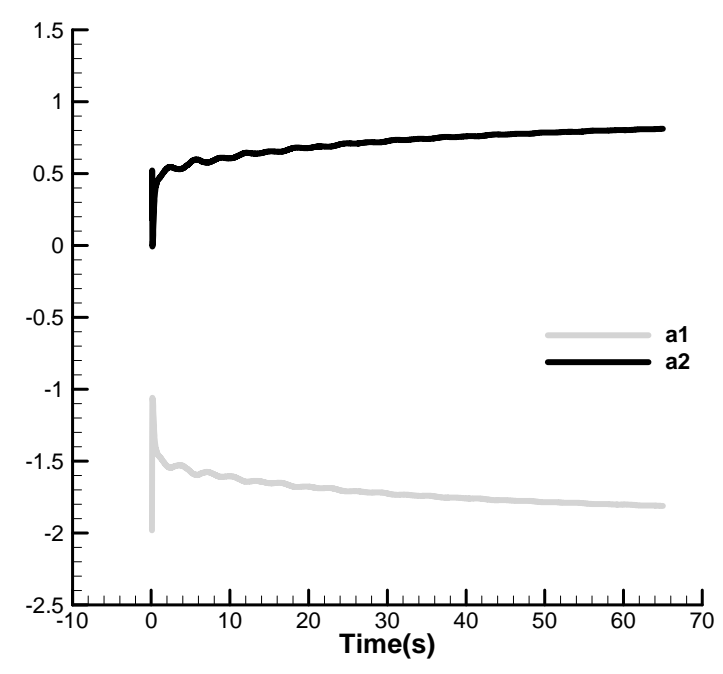

Fig. 5. Time history of $\mathrm{a}_{1}$ and $\mathrm{a}_{2}$ for pitch.

In Fig. 5, the values of $a_{1}$, and $a_{2}$ are updated in real time for pitch motion. In Fig. 6, the updated values of $b_{1}$, and $b_{2}$ are drawn in real-time.

By repeating the same algorithms for yaw and altitude, we got the Figs. 7-10 of $a_{1}, a_{2}, b_{1}$, and $b_{2}$. The parameters are updated in real-time during the simulation.

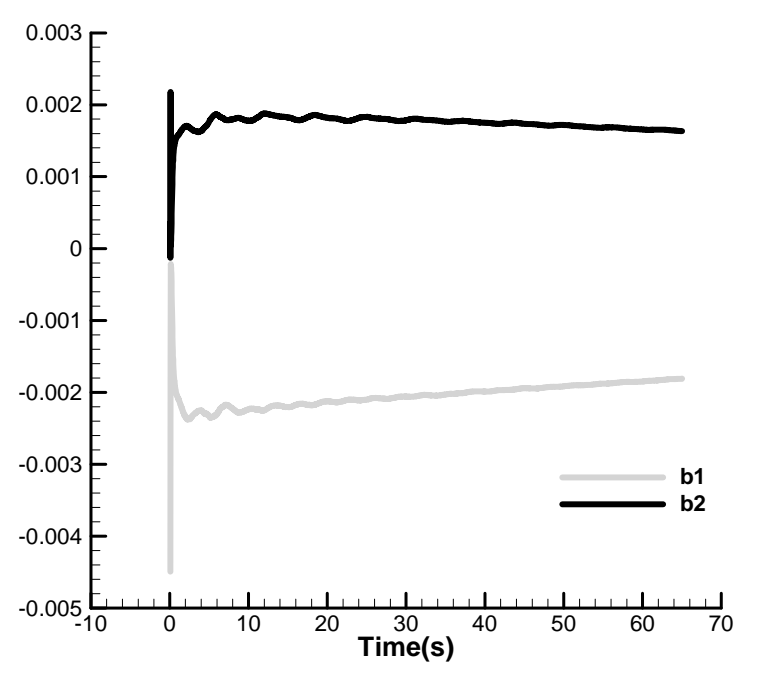

Fig. 6. Time history of $b_{1}$ and $b_{2}$ for pitch.

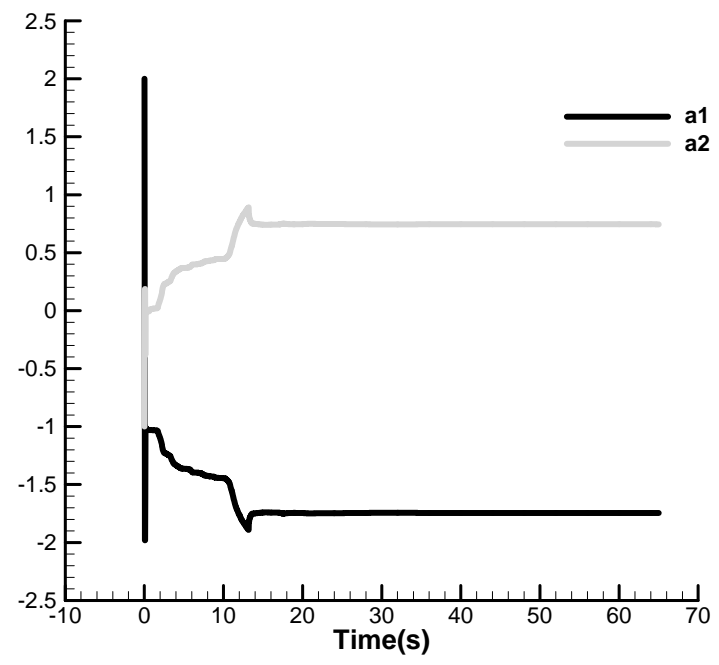

Fig. 7. Time history $\mathrm{a}_{1}$ and $\mathrm{a}_{2}$ for yaw.

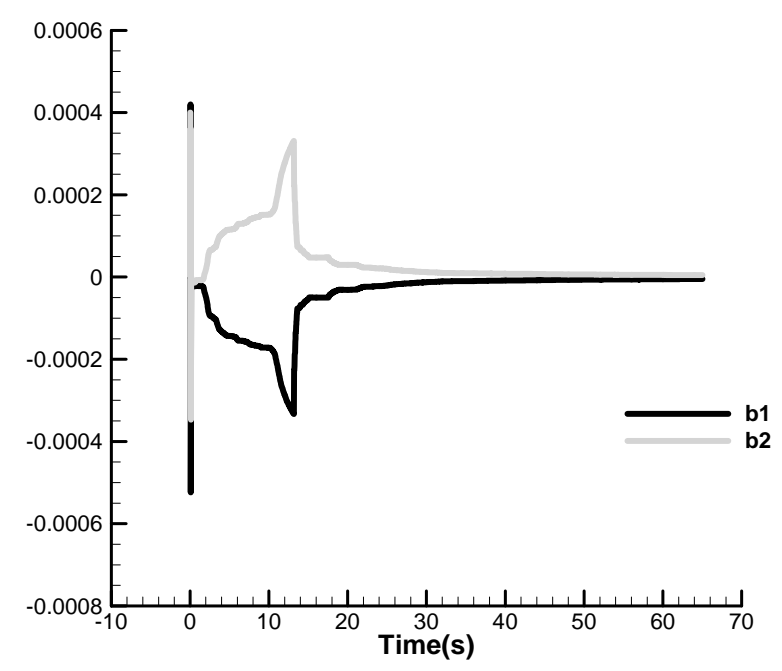

Fig. 8. Time history of $b_{1}$ and $b_{2}$ for yaw. 


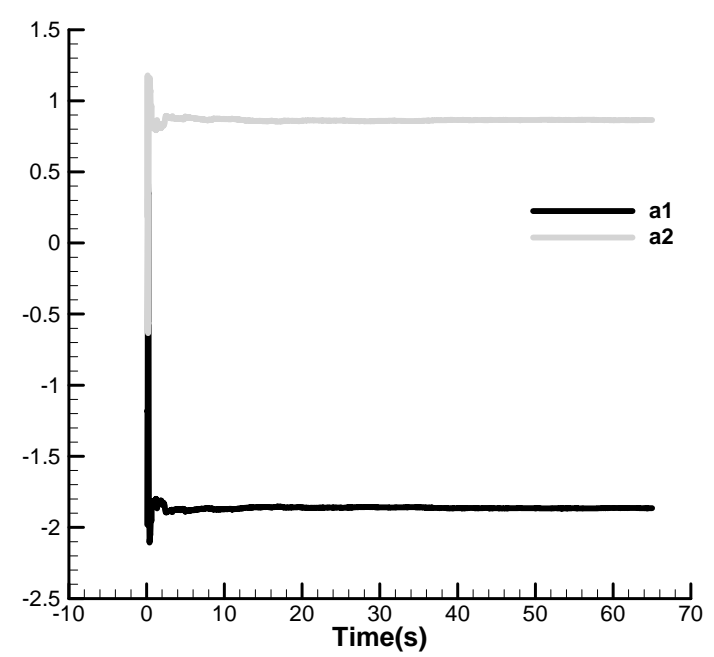

Fig. 9. Time history of $a_{1}$ and $a_{2}$ for altitude.

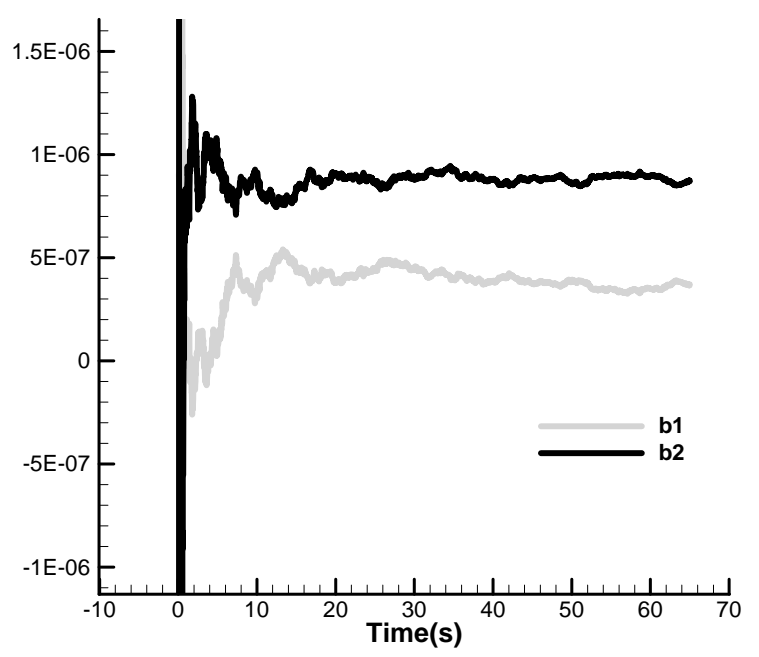

Fig.10. Time history of $b_{1}$ and $b_{2}$ for altitude.

\section{Control Design}

The control of the quad-rotor is more difficult than the fixed wing UAVs. The open loop transfer function of the quad-rotor mainly consists of double integrators which is critically stable. So the system needs aggressive control to stabilize the quad-rotor with less settling time. So the accelerator term was used with the classical control to overcome any aggressive disturbance might be occurred. It is called as a proportional-integral-derivative and acceleration PID-A controller ${ }^{12)}$.

The main objective of this algorithm is to satisfy the requirements of stability and controllability such as settling time and maximum overshoot. One application of the quad-rotor is the variable-mass system; the weight of the system is changing with time due to the variation of the tether length. Therefore, the robust control was chosen to adapt these changes in the system during flight.
From the governing equations, it's clear that the system of the quad-rotor will be represented as shown in equation (20):

$G(s)=\frac{\text { const }}{s^{2}}$

The value of the const will vary depending on roll, pitch, yaw, and altitude values as well as the mass changing. So the DC gain will be used as a constant value. Thus the controller will be designed for the given transfer function:

$$
G(s)=\frac{1}{s^{2}}
$$

So the transformation in the $\mathrm{z}$-domain will be as shown in equation (22):

$G(z)=\frac{0.0002\left(z^{-1}+z^{-2}\right)}{1-2 z^{-1}+z^{-2}}$

The control action $u$ of the motor will be multiplied by the DC gain $\mathrm{M}$ as shown $u=G_{c} M$, Then the controller parameters $\left(K_{p}, K_{i}, K_{d}, K_{a}\right)$ will be fixed and the change will be only for the DC gain $M$. The value of $M$ will be updated in real-time based on the open loop transfer function parameters. The controller action $G_{C}$ can be calculated from Equation (23) to keep the system response stable.

$$
G_{c}(t)=K_{p} e+K_{d} \dot{e}+K_{i} \int_{0}^{t} e d t+K_{a} \ddot{e}
$$

The values of $M$ are given in Figs. 11-12. These values are calculated based on the division of 0.0002 and the value of $b_{0}$.

Now the controller can stabilize the quad-rotor after connecting it with the power supply at ground. The disturbance generated from the tether will be considered in the model because the system identification is updating the model parameters in real-time. The given figures show the simulation of the take-off from altitude zero to 10 meters. The controller given in Equation (23) is applied to the attitude and altitude but the value of DC gain $M$ will be different for each degree of freedom; roll, pitch, yaw, and altitude. The values of the controller gains are given in the Table 2 .

Table 2. Control gains.

\begin{tabular}{|c|c|}
\hline Parameters (units) & Value \\
\hline $\mathrm{K}_{\mathrm{p}}\left(\mathrm{s}^{-1)}\right.$ & 90 \\
\hline $\mathrm{K}_{\mathrm{d}}(-)$ & 15 \\
\hline $\mathrm{K}_{\mathrm{i}}\left(\mathrm{s}^{-2}\right)$ & 60 \\
\hline $\mathrm{K}_{\mathrm{a}}(\mathrm{s})$ & 0.2 \\
\hline
\end{tabular}

Due to the change in the altitude, the mass of the quad-rotor will be increased. This change of the mass will be given from the Equation (24)

$m=m_{0}+0.2 h$ 
Where, $m_{0}$ is the mass of the quad-rotor without tether, and $h$ is the altitude of the quad-rotor. Therefore, the variation in the height will cause change to the open loop transfer function for roll, pitch, yaw, and altitude. Then, the controller can satisfy the required input and response depending on the motor's capability. The following Figs. 13-16 show the response for roll, pitch, yaw, and altitude due to inputs given in Equation (25-28).

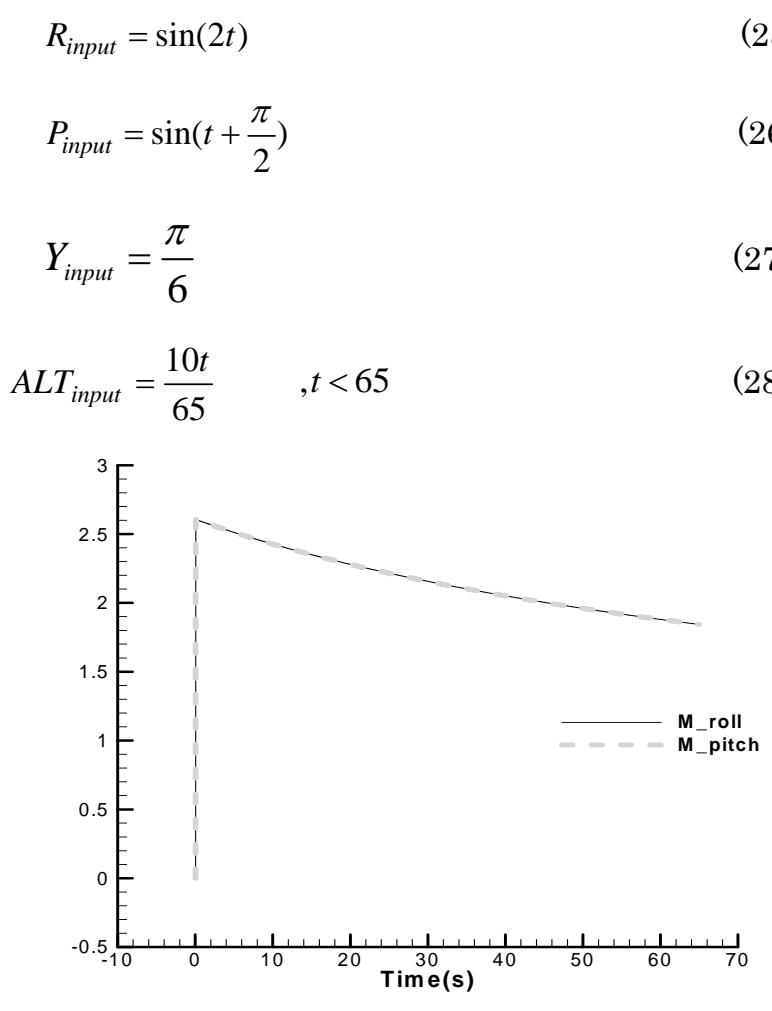

Fig. 11. Time history of the $\mathrm{M}$ value for roll and pitch.

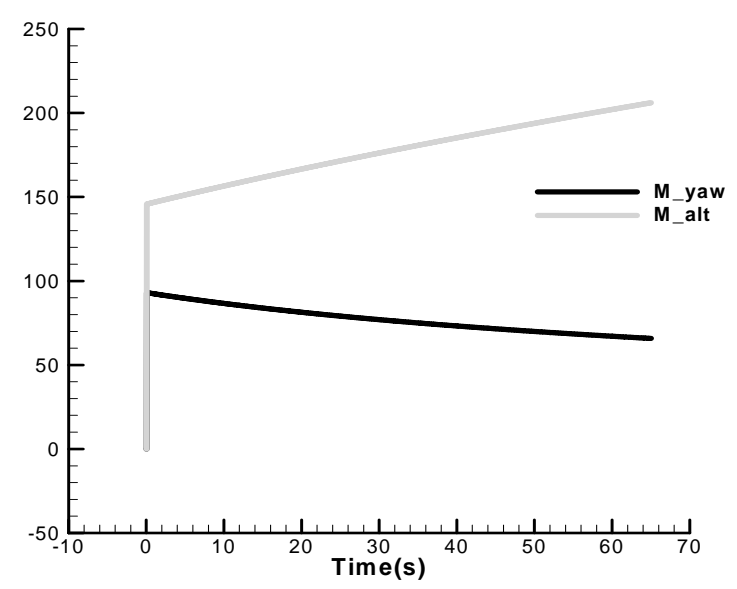

Fig. 12. Time history of the M value for yaw and altitude.

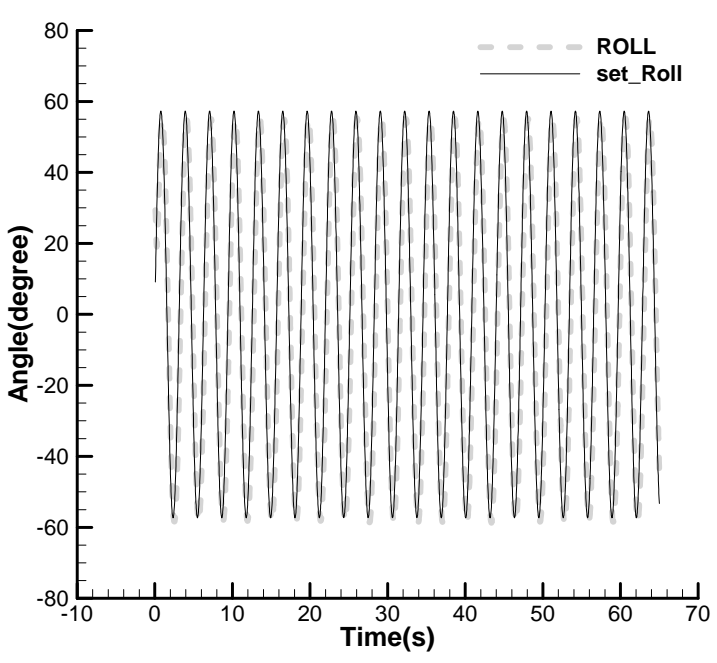

Fig. 13. Time history of the expected roll and the input.

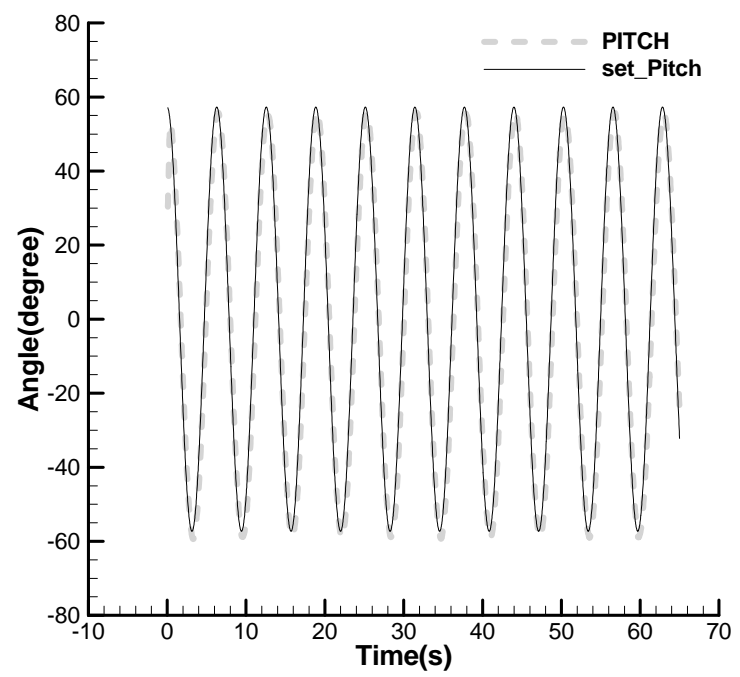

Fig. 14. Time history of the expected pitch and the input.

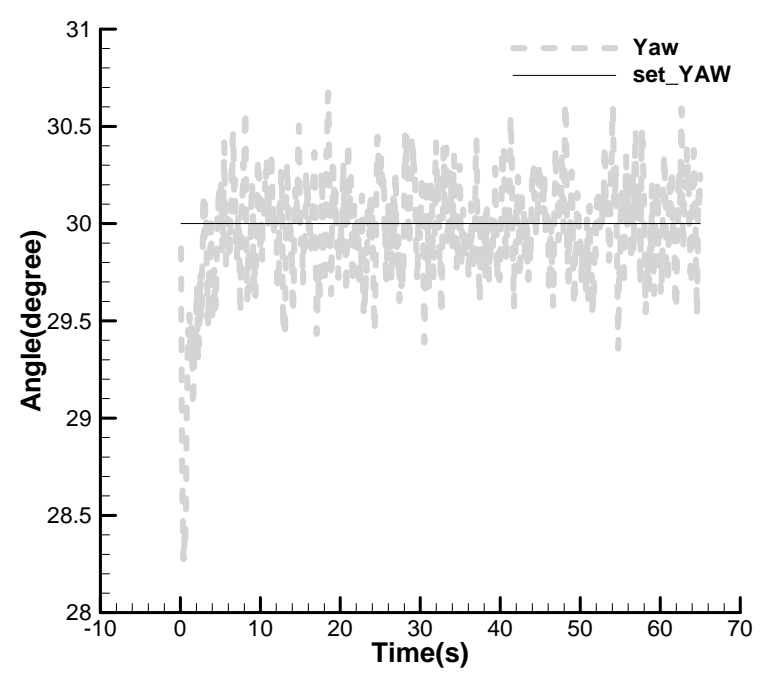

Fig. 15. Time history of the expected yaw and the input.

The set value of Fig. 15 is 30 degrees and not changing with the time because the quad rotor is required to move in certain direction. 


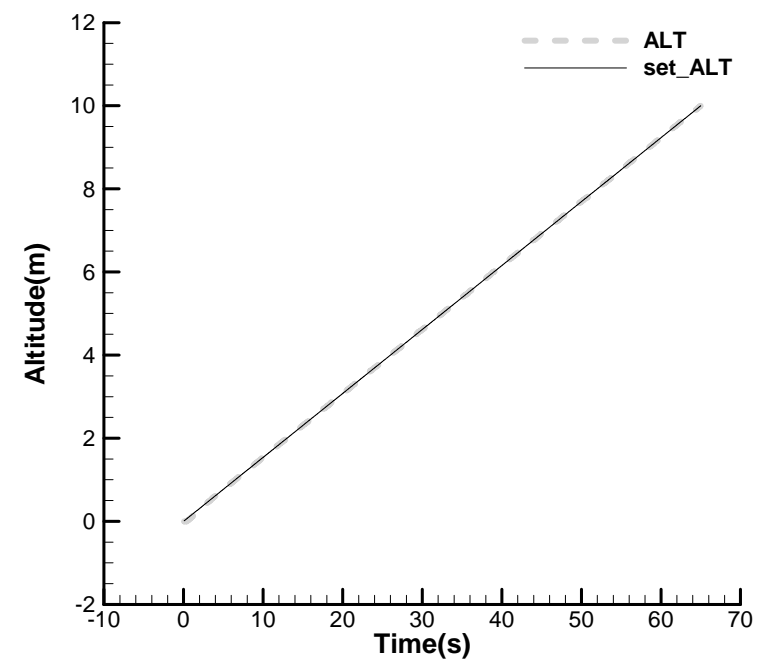

Fig. 16. Time history of the expected altitude and the input.

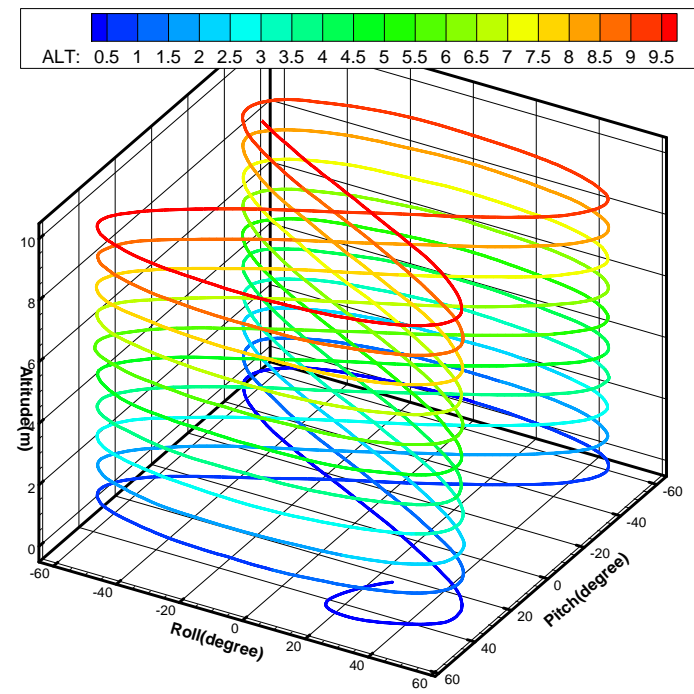

Fig. 17. Relation between roll and pith during the change of altitude from zero to 10 meters.

\section{Conclusion}

Using placket's algorithm as a system identification solves difficult problems such as the asymmetry of the motors and the variation of the system weight. So updating the parameters in real-time gives good chance to keep the quad-rotor stable regardless the flight conditions or the system failure that may happen. The system of the quad-rotor is mainly non-linear, and the linearized model that was derived is acceptable under some assumptions.

The system identification results were compared with the linearized model to check the accuracy of the system identification algorithm. Finally, this paper solved the problem of the flight time limitation using the system identification and adaptive controller in real-time considering the mass-varying of the system and the wind disturbance that might happen during flight.

\section{References}

1) Dief, T., Yoshida, S., "Review: Modeling and Classical Controller Of Quad-rotor”. International Journal of Computer Science and Information Technology \& Security (IJCSITS), ISSN: 2249-9555 Vol. 5, No4, August 2015.

2) Abdelkhalek, A., Mohamed S., Ahmed A., Dief, T., "Attitude Stability of Quadcopter Using Classic Control with Angular Acceleration." International Journal of Computer Science and Information Technology \& Security (IJCSITS), ISSN: 2249-9555 Vol. 5, No4, August 2015.

3) Dief, Tarek N., and Shigeo Yoshida. "System Identification for Quad-rotor Parameters Using Neural Network." Evergreen 3, no. 1 (2016): 6-11.

4) Deif, Tarek Naem, Ayman H. Kassem, and Gamal M. El Baioumi. "Modeling and Attitude Stabilization of Indoor Quad Rotor." International Review of Aerospace Engineering (IREASE) 7, no. 2 (2014): 43-47.

5) Dief, T. N., Yoshida, S., and Abdelhady, M. G., "Attitude and altitude stabilization of quad rotor using parameter estimation and self-tuning controller", AIAA Atmospheric Flight Mechanics Conference, June (2015), Doi: 10.2514/ 6.2015-2392.

6) Carrillo, L. R., et al. "Stabilization and trajectory tracking of a quad-rotor using vision" Journal of Intelligent \& Robotic Systems 61.1-4 (2011): 103-118.

7) Hoffmann, G. M., et al. "Quadrotor Helicopter Flight Dynamics And Control: Theory And Experiment". Proc of The AIAA Guidance, Navigation and Control Conference. Vol 2. 2007.

8) Mellinger, D., Shomin, M., and Kumar, V., "Control of Quadrotors for Robust Perching and Landing.pdf," Int. Powered Lift Conf., pp. 119-126, 2010.

9) Hehn, M. and D’Andrea,R. , “A flying inverted pendulum,” Proc. - IEEE Int. Conf. Robot. Autonomous. No. 2, pp. 763-770, 2011.

10) Deng, F., Zhao, Y., Tang, X., "Tension analysis of elastic rope linked with balloon in air under wind force”, Journal of Northeast Institute of Heavy Machinery, vol.I5, no.02, pp.176-180, 1991.

11) Shi, X., Li Y., Shi, H., "Dynamic Simulation of Ascending Tethered Balloon”, Acta Aeronautica et Astronautica Sinica, vo1.30 no,04, pp,609-613, 2009. 
12) Li, Y., Xie, Q., and Han, J., "Modeling and PID control of tethered unmanned quadrotor helicopter." Mechatronic Sciences, Electric Engineering and Computer (MEC), Proceedings 2013 International Conference on. IEEE, 2013.

13) Sergei, L., and D'Andrea, R., "Stabilization of a flying vehicle on a taut tether using inertial sensing." 2013 IEEE/RSJ International Conference on Intelligent Robots and Systems. IEEE, 2013.

14) Abdelkhalek, M. A., El-Demerdash, M. S., El-Tahan, A. A., \& Dief, T. N., "Attitude Stability of Quadcopter Using Classic Control with Angular Acceleration”. IRACST - International Journal of Computer Science and Information Technology \& Security (IJCSITS), ISSN: 2249-9555 Vol. 5, No4, August 2015.

15) Dutton, K., Thompson, S., and Barraclough, B., The art of control engineering. Addison-Wesley Longman Publishing Co., Inc., 1997.

Huang, H., Hoffmann , G. M., Waslander, S. L.,

16) Tomlin, C. J. "Aerodynamic And Control Of Autonoumous Maneuvering." Robotics And Automation, 2009. ICRA'09.IEEE Interntaional Conference On .IEEE, 2009.

17) Lupashin, S., D'Andrea, R., "Stabilizaiton of a flying vehicle on a taut tether using inertial sensing," in Proceedings of the IEEE International Conference on Intelligent Robots and Systems, 2013, pp. 2432-2438.

18) Nicorta, M., Naldi, N., Garone, E., "Taut cable control of a tethered UAV," in Proceedings of the IFAC World Congress, 2014.

19) Tognon, M., Franchi, A., "Nonlinear observer-based tracking control of link stress and elevation for a tethered aerial robot using inertial measurements," in Proceedings of the IEEE International Conference on Robotics and Automation, 2015. 